\title{
Metal nanoparticle doped coloured coatings on glasses and plastics through tuning of surface plasmon band position
}

\author{
GOUTAM DE*, SAMAR KUMAR MEDDA, SUCHETA DE and SUDIPTO PAL \\ Sol-Gel Division, Central Glass and Ceramic Research Institute, Kolkata 700 032, India
}

\begin{abstract}
Several noble metal nanoparticles doped sol-gel derived thin coloured films have been synthesized and characterized. These are pure ( $\mathrm{Ag}, \mathrm{Au}, \mathrm{Cu}$ and $\mathrm{Pt}$ ), mixed/alloy (Ag-Cu, $\mathrm{Au}-\mathrm{Cu}, \mathrm{Au}-\mathrm{Ag}$ and $\mathrm{Au}-\mathrm{Pt}$ ) nanoparticles in $\mathrm{SiO}_{2}$, Au in mixed $\mathrm{SiO}_{2}-\mathrm{TiO}_{2}$ and $\mathrm{SiO}_{2}-\mathrm{ZrO}_{2}$, Au and Ag nanoparticles in inorganic-organic hybrid film matrices etc. This investigation leads to the development of tailor-made coloured coatings by tuning the surface plasmon resonance (SPR) band positions originating from the embedded nanometals by controlling mainly (i) refractive index of the film matrices and (ii) nanoalloy composition. In the later case a new layerby-layer (two-layer) synthetic protocol has been developed to prepare binary nanoalloy particles with controlled atomic ratios.
\end{abstract}

Keywords. Metal (single/mixed/alloy) nanoparticles; surface plasmon; coloured coatings on glasses and plastics; layer-by-layer coatings by sol-gel; refractive index tuning; NLO material.

\section{Introduction}

Noble metal nanoparticles (mainly $\mathrm{Au}, \mathrm{Ag}$ and $\mathrm{Cu}$ ) have been studied extensively because of their unique linear (Sun and Xia 2003; Medda et al 2005a; Pal and De 2005; Jain et al 2006; Liz-Marzán 2006) and nonlinear optical properties (De et al 1996; Kiran et al 2004; Venkatram et al 2006). These unique properties are associated with their strong surface plasmon resonance (SPR), which are due to $d-d$ transitions pushed into the visible part of the spectrum. As a consequence they show very intense colouration and their optical properties depend strongly upon the particle size and shape, concentration, refractive index of the surrounding medium (embedding matrix), interparticle interactions, coupling and mixing of plasmon bands due to alloying of different metal nanoparticles etc (see, for example, Liz-Marzán 2006). Using these metal nanoparticles as a colouring source we have undertaken a research programme to develop several colour coatings applicable to glass and plastic substrates (Medda et al 2005a, b; Pal and De 2005; De and De 2006a; Pal and De 2007a). The manufacturing process involves preparation of sols using suitable metal organic precursors (e.g. alkoxides or functionalized alkoxides also known as inorganic-organic hybrid precursors) and soluble single or mixture of noble metal salts. The sols after controlled hydrolysis-condensation reactions could be applied to glass or plastic substrates by usual dip- or spin-coating technique. The as-prepared coatings are first dried and then heat-treated at $450^{\circ} \mathrm{C}$ and above (in case of glass) in di-

*Author for correspondence (gde@ cgcri.res.in) fferent gas atmospheres or cured photochemically through UV light (in case of plastic substrates). The heat treatment causes densification of the matrix as well as reduction of metal salts to the corresponding metal nanoparticles. On the other hand, UV curing causes polymerization of organic functionalities and inorganic networks, and reduction of metal salts simultaneously. In this programme, the SPR absorption in the visible spectrum of light (which causes intense colouration) arising from the embedded nanometals was tuned by controlling the following: (i) refractive index of the coating matrix, (ii) nanoalloy type and atomic ratio (pre-determined) and (iii) composition of nanoalloys formed by interlayer diffusion using a new two-layer (TL) approach (Pal and De 2005, 2007). In case of (i), we tried to keep the shape and size of nanoparticles similar in coating matrices having different refractive index values. The refractive indices are controlled by changing the ratios of two dielectrics (e.g. $\mathrm{SiO}_{2}$ and $\mathrm{TiO}_{2}$ or $\mathrm{ZrO}_{2}$ ). In case of (ii) and (iii), so far $\mathrm{SiO}_{2}$ has been used as the matrix. The different noble metal soluble salts (e.g. gold and copper chloride, silver and copper nitrate, gold and platinum chloride) in fixed ratios (pre-determined) are mixed with matrix-precursors to obtain the desired alloy/mixed nanoparticles. In case of (iii), a layer-by-layer (two-layer) deposition technique was employed to obtain tunable alloy nanoparticles. Here two successive overlapping individual layers containing different metal ion doped sols, respectively are deposited on glass substrates. These two-layer (TL) film assemblies when treated thermally/photochemically, interlayer diffusion of nanometal(s) causes alloying in a controlled way. The concept lying behind this new approach is described below. The synthesis of different binary alloys (e.g. $\mathrm{Au}-\mathrm{Cu}$ (De et al 
2000; De and Rao 2003), Co-Ni (Mattei et al 2002) and $\mathrm{Au}-\mathrm{Pt}$ (De and Rao 2005)) and mixed (e. g. Ag-Cu (De 1998)) nanoparticle doped coatings from sols obtained by simply mixing soluble nitrate or chloride salts into TEOS-derived silica sols followed by thermal annealing could be achieved using method (ii). However, this simple approach failed in the case of systems like $\mathrm{Au}-\mathrm{Ag}$ because of non availability of Au- and Ag-salts remaining soluble in a common solvent. Thus in case of $\mathrm{Au}-\mathrm{Ag}$, a new TL protocol has been designed (Pal and De 2005). This approach works extremely well in obtaining films containing uniform $\mathrm{Au}-\mathrm{Ag}$ nanoalloys with controlled atomic ratios. This new approach also works in other cases (e.g. $\mathrm{Au}-\mathrm{Cu}(\mathrm{Pal}$ and $\mathrm{De} 2007 \mathrm{~b})$ ) where soluble salts are available.

In this article, our work related to the development of coloured coatings on glass and plastic substrates will be reviewed and presented in brief. Apart from the linear optical properties (colour), the nonlinear optical (NLO) properties are also of great interest for application of metal nanoparticle doped coatings in ultra fast optical switches. Some results will be highlighted at the end as references (Kiran et al 2004; Venkatram et al 2006).

\section{Experimental}

The individual metal $(\mathrm{Au}, \mathrm{Cu}, \mathrm{Ag}$ and $\mathrm{Pt})$ nanoparticle doped coatings were prepared from $\mathrm{SiO}_{2}$ and $\mathrm{SiO}_{2}-\mathrm{TiO}_{2}$ hybrid sols using silicon, titanium, zirconium and substituted (functionalized) silicon alkoxides, $\mathrm{HAuCl}_{4} \cdot 3 \mathrm{H}_{2} \mathrm{O}$, $\mathrm{Cu}\left(\mathrm{NO}_{3}\right)_{2}, \mathrm{AgNO}_{3}, \mathrm{H}_{2} \mathrm{PtCl}_{6}$, acetylacetone (acac), n-propanol, $n$-butanol, $i$-butanol and acidulated water. In case of mixed (binary) metal nanoparticles doped sols two soluble metal salts are incorporated into the sols in suitable proportions for desired alloy compositions. Coatings could be prepared either by the sol-gel dip- or spin-coating techniques followed by thermal annealing and/or UV curing. To control the matrix refractive index of $\mathrm{SiO}_{2}: \mathrm{TiO}_{2}$ and $\mathrm{SiO}_{2}: \mathrm{ZrO}_{2}$ molar ratios (nominal) were varied from $1: 0$ to about $1: 4$. In case of $\mathrm{SiO}_{2}-\mathrm{TiO}_{2}$ system the oven dried $\left(90^{\circ} \mathrm{C}\right)$ films were directly heated to $500^{\circ} \mathrm{C}$ in air to obtain the final dense films, whereas for $\mathrm{SiO}_{2}-\mathrm{ZrO}_{2}$ system after oven drying a UV curing step was given before proceeding to further heat-treatment. The molar ratio of the metal to equivalent oxide $\left(\mathrm{SiO}_{2} / \mathrm{SiO}_{2}-\mathrm{TiO}_{2} / \mathrm{SiO}_{2}-\mathrm{ZrO}_{2}\right)$ was kept close to 3 equivalent mol\% metal-97\% oxide. In cases of inorganic-organic composite coatings applicable for plastic substrates, about 2.2-3.2 equivalent $\mathrm{mol} \%$ metal was incorporated into the hybrid matrix $\left(\mathrm{SiO}_{2}\right.$ polyethylene oxide $\left.(\mathrm{PEO}) / \mathrm{SiO}_{2}-\mathrm{PEO}-\mathrm{TiO}_{2}\right)$. Coatings were deposited on clean soda-lime silica, silica glass and polycarbonate substrates by dip-coating employing a lifting speed of 2-4 inches $/ \mathrm{min}$. The resulting films deposited on glass substrates were dried at $60^{\circ} \mathrm{C}$ for $60 \mathrm{~min}$ followed by annealing at $450^{\circ} \mathrm{C}$ for $30 \mathrm{~min}$ in air for the decompo- sition of organics. Subsequent heat-treatments in controlled atmospheres were then followed. The maximum annealing temperature and gas atmospheres were varied in different systems. In case of two-layer coatings, the second layer has been deposited onto the first layer after drying at $60^{\circ} \mathrm{C}$. The TL coating assembly was finally dried at $60^{\circ} \mathrm{C}$ for $30 \mathrm{~min}$ and subjected to UV and necessary thermal treatments. The coatings deposited on PC were first dried at $90^{\circ} \mathrm{C}$ followed by UV curing using a conveyorized UV curing machine. The coating thickness, estimated by a profilometer, was in the $200-1000 \mathrm{~nm}$ range. Undoped coatings were also prepared for the measurement of matrix refractive index values. The details of characterization techniques employed (e.g. measurements of coatings, thickness, refractive index, FTIR, UV-visible, XRD, TEM, energy dispersive X-ray analysis etc) in these studies were described elsewhere (Pal and De 2005; Medda et al 2005a).

\section{Results and discussion}

\subsection{Refractive index controlled plasmon tuning}

The SPR absorption position of nanometals was tailored by controlling the refractive index of the embedding matrix (Medda et al 2005a; De and De 2006b; De et al 2008). The optical absorption of metal nanoparticles is dependent mainly on concentration, size, shape and the nature of the surrounding medium. Among these the concentration is related to the intensity of absorption peaks. Therefore, if the concentration, size and shape of the nanoparticles can be kept similar then the influence of surrounding medium could be realized. In fact, the influence of the non absorbing surrounding medium (here coating matrix) is usually related to its refractive index (Mulvaney 1996; Liz-Marzán 2006), since Mie theory predicts resonance to occur when $\varepsilon_{1}(\omega)=-2 \varepsilon_{\mathrm{m}}$, where $\varepsilon_{1}(\omega)$ and $\varepsilon_{\mathrm{m}}$ are the real components of the metal dielectric function at angular frequency, $\omega$ and medium dielectric constant, respectively. Therefore, if the size- and shape effects of the nanoparticles are not considered, the position of SPR arising from the metal nanoparticles can be tailored by changing the refractive index of the embedding matrices. Using this idea, different coloured coatings on ordinary sheet glasses were prepared by incorporating $\mathrm{Au}$ nanoparticles in $\mathrm{SiO}_{2}-\mathrm{TiO}_{2}$ and $\mathrm{SiO}_{2}-\mathrm{ZrO}_{2}$ matrices having different refractive index values ranging from about 1.41-1.94. As for example the $\mathrm{Au}-\mathrm{SPR}$ can be tuned from $540 \mathrm{~nm}$ to $\sim 600 \mathrm{~nm}$ on going from $\mathrm{SiO}_{2}$ to $\mathrm{SiO}_{2}-\mathrm{TiO}_{2}(1: 3)$ matrix, and as a consequence, different coloured coatings (pink, magenta, violet, royal-blue and blue) were obtained (Medda et al 2005a). Similar effects are also observed in case of Au in $\mathrm{SiO}_{2}-\mathrm{ZrO}_{2}$ system (De et al 2008). In this case also the red-shifting of Au-SPR from $545-592 \mathrm{~nm}$ is clearly observed on going from $\mathrm{SiO}_{2}: \mathrm{ZrO}_{2}=1: 0$ to $1: 4$ due to the 
Table 1. Au-SPR peak positions with respect to the refractive index of the embedding $\mathrm{SiO}_{2}$ : $\mathrm{TiO}_{2}$ film matrix (part of data presented in this table has been reproduced from Medda et al 2005a).

\begin{tabular}{lcccc}
\hline & $\begin{array}{c}\text { Molar ratio (nominal) of } \\
\mathrm{SiO}_{2}: \mathrm{TiO}_{2}\end{array}$ & Annealing condition & $\begin{array}{c}\text { Refractive index* } \\
\left(500^{\circ} \mathrm{C}\right)\end{array}$ & $\begin{array}{c}\text { Au-SPR position (nm) } \\
\left(500^{\circ} \mathrm{C}\right)\end{array}$ \\
\hline & $1: 0$ & $90^{\circ} \mathrm{C} / 500^{\circ} \mathrm{C}$ & 1.411 & 542 \\
$\mathrm{SiO}_{2}-\mathrm{TiO}_{2}$ series & $1: 0 \cdot 43$ & Do & 1.604 & 555 \\
& $1: 1$ & Do & 1.713 & 572 \\
& $1: 1.5$ & Do & 1.862 & 580 \\
& $1: 2 \cdot 33$ & Do & 1.913 & 588 \\
& $1: 3$ & Do & 1.939 & 600 \\
\hline
\end{tabular}

*Refractive index values were measured from the undoped films

Table 2. Au-SPR peak positions with respect to the refractive index of the embedding $\mathrm{SiO}_{2}: \mathrm{ZrO}_{2}$ film matrix (part of data presented in this table has been reproduced from De et al 2008).

\begin{tabular}{lcccc}
\hline & $\begin{array}{c}\text { Molar ratio (nominal) of } \\
\mathrm{SiO}_{2}: \mathrm{ZrO}_{2}\end{array}$ & Annealing condition & $\begin{array}{c}\text { Refractive index* } \\
\left(500^{\circ} \mathrm{C}\right)\end{array}$ & $\begin{array}{c}\text { Au-SPR position (nm) } \\
\left(500^{\circ} \mathrm{C}\right)\end{array}$ \\
\hline & $1: 0$ & $90^{\circ} \mathrm{C} / \mathrm{UV} / 500^{\circ} \mathrm{C}$ & 1.410 & 546 \\
$\mathrm{SiO}_{2}-\mathrm{ZrO}_{2}$ series & $1: 1$ & Do & 1.698 & 558 \\
& $1: 1 \cdot 5$ & Do & 1.728 & 571 \\
& $1: 2 \cdot 33$ & Do & 1.765 & 581 \\
\hline
\end{tabular}

*Refractive index values were measured from the undoped films
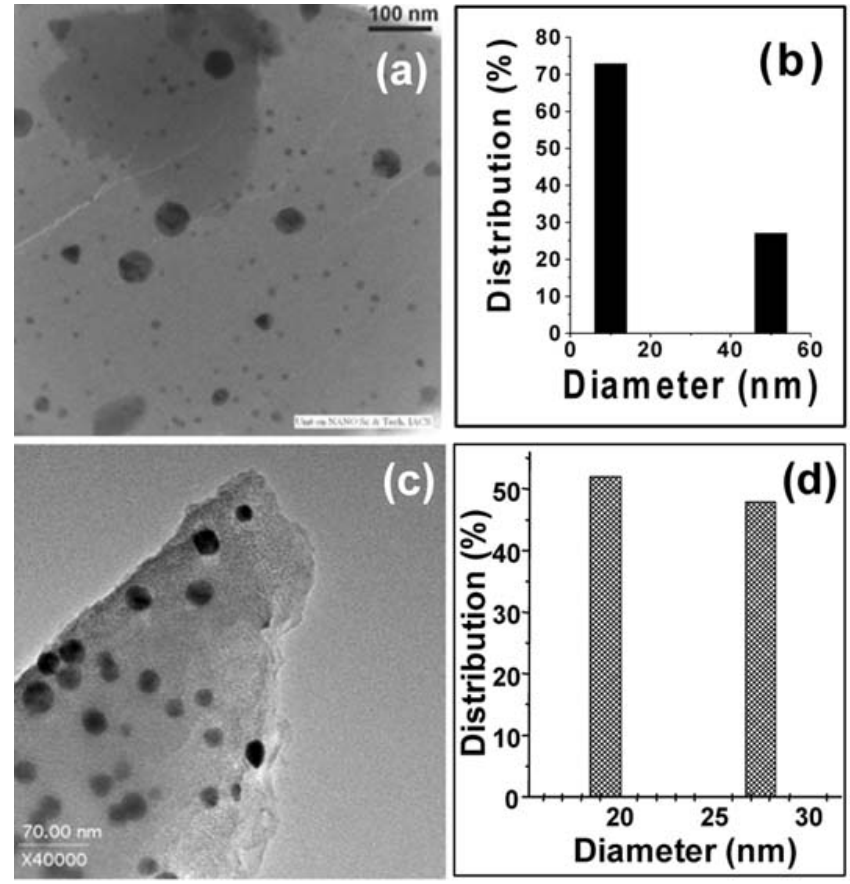

Figure 1. TEM of Au nanoparticle doped films obtained after final heat-treatment at $500^{\circ} \mathrm{C}$. Image and corresponding size distributions of $\mathrm{Au}$ nanometals embedded in matrices $\mathrm{SiO}_{2}$ : $\mathrm{ZrO}_{2}(1: 4)$, marked as (a) and (b) and $\mathrm{SiO}_{2}: \mathrm{TiO}_{2}(2: 3)$, marked as (c) and (d)

systematic increase of refractive index of the matrices after heat-treatment at $500^{\circ} \mathrm{C}$. Tables 1 and 2 show the
SPR absorption peak positions of the final heat-treated $\left(500^{\circ} \mathrm{C}\right)$ coatings arising from the embedded Au nanoparticles in refractive index controlled $\mathrm{SiO}_{2}-\mathrm{TiO}_{2}$ and $\mathrm{SiO}_{2}-$ $\mathrm{ZrO}_{2}$ film matrices, respectively. The corresponding refractive index values of the undoped films are also shown in tables 1 and 2. Clearly, the Au SPR is systematically red-shifted with increasing refractive index of coating matrices. It may be pointed out here that the coatings are transparent and spot-free and sufficiently hard for any kind of practical applications. Furthermore as the extinction coefficient of such SPR absorptions are very high, small amount of doping of such nanometals causes intense colouration.

To check the shape and size of the Au nanoparticles, TEM and XRD studies were undertaken. We found similar shape and size distributions of $\mathrm{Au}$ nanoparticles embedded in respective series of $\mathrm{SiO}_{2}-\mathrm{TiO}_{2}$ and $\mathrm{SiO}_{2}-\mathrm{ZrO}_{2}$ systems. In case of $\mathrm{SiO}_{2}-\mathrm{TiO}_{2}$ series presence of spherical $\mathrm{Au}$ nanoparticles of an average diameter of $20 \pm 3 \mathrm{~nm}$ has been observed whereas in the $\mathrm{SiO}_{2}-\mathrm{ZrO}_{2}$ system $\mathrm{Au}$ nanoparticles having diameter of about $10 \mathrm{~nm}$ and a few bigger $\mathrm{Au}$ nanoplates of hexagonal and triangular shapes are found to co-exist. The generation of these Au nanoplates most probably originated from the UV-photo reduction of $\mathrm{HAuCl}_{4}$ in the inorganic-organic hybrid matrix because earlier reports showed UV decomposition of $\mathrm{HAuCl}_{4}$ favours generation of Au nanotriangles and hexagons in solution (Zhou et al 1999). The $10 \mathrm{~nm}$ particles which are present in $>70 \%$ population are expected to dominate the optical features in case of $\mathrm{Au} / \mathrm{SiO}_{2}-\mathrm{ZrO}_{2}$ 
films. As the shape and size distributions of Au nanoparticles are different we observed some anomalies in the SPR peak positions of the two series of final heat-treated film samples (tables 1 and 2). As for example the refractive index values of $\mathrm{SiO}_{2}: \mathrm{TiO}_{2}(1: 1 \cdot 5)$ and $\mathrm{SiO}_{2}: \mathrm{ZrO}_{2}(1: 4)$ films are comparable, however, the $\mathrm{Au}-\mathrm{SPR}$ positions are observed in different positions at 580 and $592 \mathrm{~nm}$, respectively. This difference could be due to the presence of nanoplates (anisotropic) in the $\mathrm{SiO}_{2}: \mathrm{ZrO}_{2}$ films which usually pushed the SPR towards higher wavelengths. The XRD of $\mathrm{Au} / \mathrm{SiO}_{2}-\mathrm{TiO}_{2}$ films showed weak broad peaks corresponding to $f c c \mathrm{Au}$ nanometals. On the other hand, $\mathrm{Au} / \mathrm{SiO}_{2}-\mathrm{ZrO}_{2}$ films showed relatively strong peaks corresponding to the $\mathrm{Au}$ (111) and $\mathrm{Au}$ (222) reflections only. This observation supports the presence of $2 \mathrm{D} \mathrm{Au}$ nano-
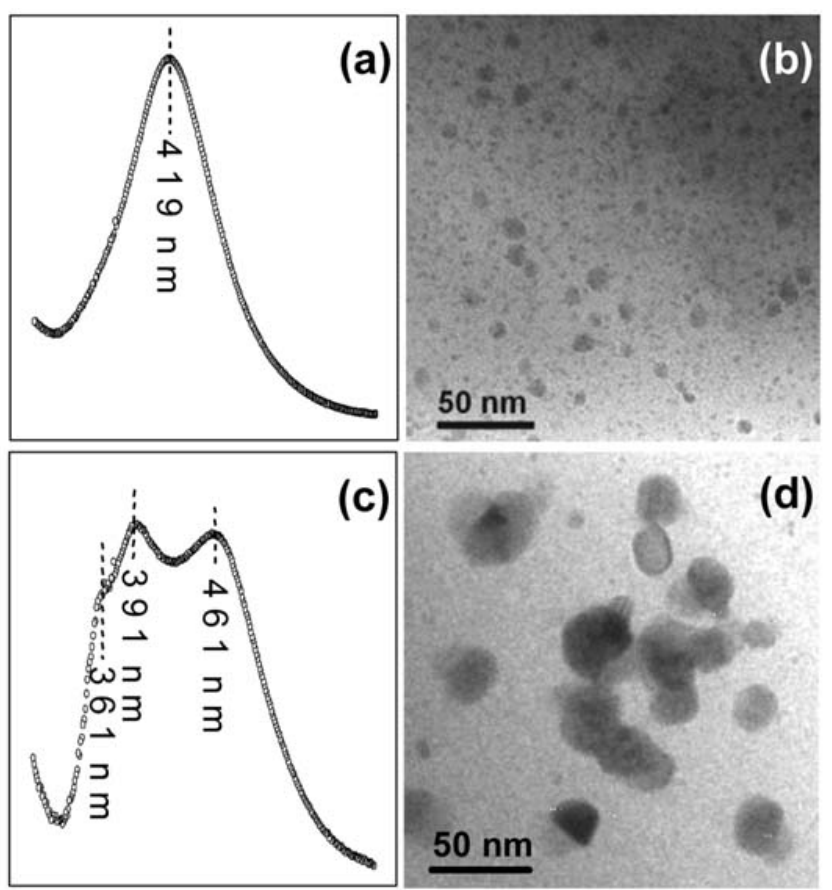

(c)

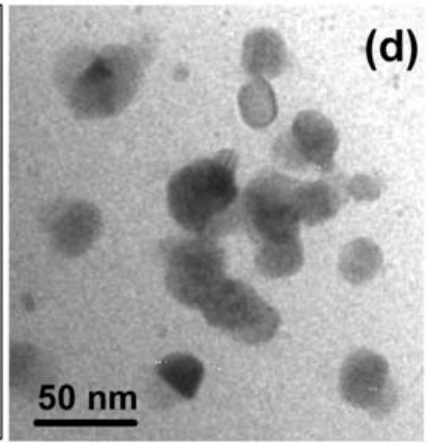

Figure 2. Optical absorptions and the corresponding TEM images of $\mathrm{Ag}$ nanoparticle doped $\mathrm{SiO}_{2}-\mathrm{PEO}$ hybrid films after UV irradiations with energies $5.3 \mathrm{~J} / \mathrm{cm}^{2}$ (a) and (b);31.8 J/ $\mathrm{cm}^{2}$ (c) and (d). (The curves shown in (a) and (c) are reproduced from De and De 2006a).

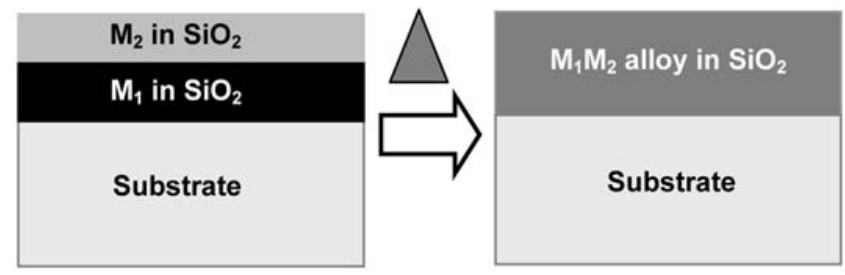

Figure 3. Schematic representation of the $2 \mathrm{~L}$ protocol used for the generation of alloy nanoparticles in controlled atomic ratios inside the film matrices. plates in $\mathrm{Au} / \mathrm{SiO}_{2}-\mathrm{ZrO}_{2}$ films. The difference in size and shape of Au nanoparticles in the two series could be due to the UV treatment step given prior to heat-treatment in case of $\mathrm{SiO}_{2}-\mathrm{ZrO}_{2}$ system. In this series, the UV treatment step prior to thermal treatment was essential for the generation of $\mathrm{Au}$ nanoparticles and decomposition of $\mathrm{Zr}$-acac linkages. These UV treated films when heat-treated at $500^{\circ} \mathrm{C}$ produced uniform and transparent coloured coatings on glass substrates. Figure 1 shows TEM of two representative final heat-treated $\mathrm{Au} / \mathrm{SiO}_{2}-\mathrm{TiO}_{2}(2: 3)$ and $\mathrm{Au} / \mathrm{SiO}_{2}-$ $\mathrm{ZrO}_{2}(1: 4)$ samples along with the size distributions of embedded Au nanoparticles.

In another development, in situ generation of Ag nanoparticles in the refractive index controlled inorganicorganic hybrid host $\left(\mathrm{SiO}_{2}-\mathrm{TiO}_{2}-\mathrm{PEO}\right)$ leads to the formation of abrasion resistant coloured coatings (yellow to pink) on plastic substrates (Medda et al 2005b). In this case Ag nanoparticle incorporated inorganic-organic hybrid $\mathrm{SiO}_{2}-\mathrm{TiO}_{2}$ films were prepared from sols derived from tetraethyl orthosilicate, 3-(glycidoxypropyl)-trimethoxysilane, titanium isopropoxide and silver nitrate following a sol-gel dip-coating method. Hydrolysis-condensation of alkoxy groups and polymerization of epoxy groups formed the inorganic $\left(\mathrm{SiO}_{2} / \mathrm{TiO}_{2}\right)$ and polyethylene oxide (PEO) network, respectively. These films were cured thermally $\left(\sim 100^{\circ} \mathrm{C}\right)$ and photochemically using a conveyorized UV curing machine. In this system by controlling the $\mathrm{SiO}_{2} /$ $\mathrm{TiO}_{2}$ molar ratio the matrix refractive index can be tuned from 1.475 to 1.71 . Accordingly, the Ag-SPR has been shifted from about $420 \mathrm{~nm}$ (refractive index, 1.475) to about $500 \mathrm{~nm}$ (refractive index, 1.71). As a result the colour of the film can be tuned to any colour arising from

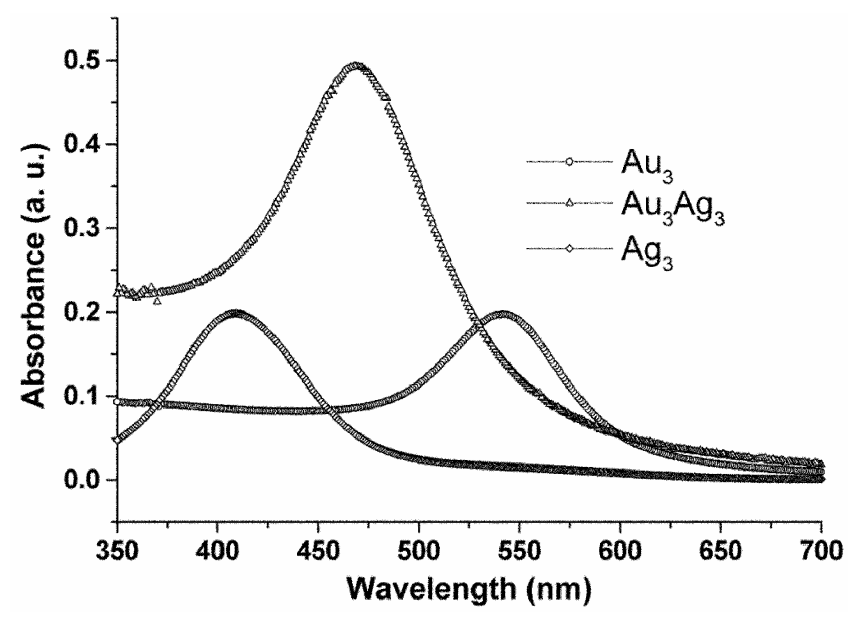

Figure 4. UV-visible spectra showing the SPR arising from $\mathrm{Au}-\mathrm{Ag}(1: 1)$ alloy nanoparticles obtained using the new $2 \mathrm{~L}$ approach. The SPR of the corresponding Au- and Ag-nanoparticle doped single-layer films are also shown. The composition of the films is 3 equivalent mol\% metal- $97 \% \mathrm{SiO}_{2}$ and accordingly the films are marked as $\mathrm{Au}_{3}, \mathrm{Au}_{3} \mathrm{Ag}_{3}$ and $\mathrm{Ag}_{3}$ in the body of the figure. (The curves shown in this figure are reproduced from Pal and De 2005). 
absorptions in the range of 420-500 $\mathrm{nm}$. TEM studies of this series show presence of very small Ag nanoparticles of size ranges $3-5 \mathrm{~nm}$. The adhesion (5B as per ASTM D3359) and abrasion resistant (pencil hardness value, 3-4H as per ASTM D3363 standard) qualities of these hybrid films are very good and can, therefore, be applicable as scratch resistant coloured coatings on transparent polycarbonate substrates.

We have also noticed controlled coarsening phenomena of $\mathrm{Ag}$ nanoparticles in $\mathrm{SiO}_{2}-\mathrm{PEO}$ hybrid matrix by $\mathrm{UV}$ light of different energies (De and De 2006a). A single surface plasmon resonance (SPR) band due to Ag nanoparticles has been observed at $419 \mathrm{~nm}$ when the Ag nanoparticles doped $\mathrm{SiO}_{2}-\mathrm{PEO}$ film is cured at $90^{\circ} \mathrm{C}$ and $\mathrm{UV}$ treated with energy, $5.3 \mathrm{~J} / \mathrm{cm}^{2}$ (figure $2 \mathrm{a}$ ). The corresponding TEM image (figure $2 \mathrm{~b}$ ) shows presence of $\mathrm{Ag}$ nanoparticles of mainly $3-4 \mathrm{~nm}(90-95 \%)$ in size. A few (5-10\%) bigger nanoparticles of size ranges $10-25 \mathrm{~nm}$ are also visible. The Ag-SPR band is gradually split and finally showed three peaks at 361 (shoulder), 391 and $461 \mathrm{~nm}$ upon increasing the UV treatment energy up to $31.8 \mathrm{~J} / \mathrm{cm}^{2}$ (figure $2 \mathrm{c}$ ). In this case the TEM study shows (figure 2d) presence of larger Ag nanoparticles of different shapes (spheroidal, elongated and fractal type small aggregates). Therefore, the modification of optical spectra of Ag nanoparticles occurred due to the coarsening of small Ag nanoparticles to the large spheroidal, elongated and fractal type small aggregates after UV treatment. The splitting of Ag-SPR band is found to be consistent with the average aspect ratio (calculated from TEM image) of Ag nanometals of anisotropic shapes (De and De 2006a). Using this simple approach the coarsening of Ag nanoparticles could be controlled by controlling the UV treatment energies.

Tuning of Au SPR band has also been accomplished using the inorganic-organic hybrid film matrix like $\mathrm{SiO}_{2}-$ $\mathrm{TiO}_{2}-\mathrm{PEO}$ (equivalent $\mathrm{SiO}_{2}: \mathrm{TiO}_{2}=2: 3$ ). In this case the refractive index of the hybrid matrix can be controlled with respect to the UV irradiation energies (De and De 2006b). As a consequence the Au-SPR has been redshifted with increasing refractive index of the matrix. The study reveals tunable coloured coatings (pink to blueviolet) when applied on glass as well as plastic substrates.

\subsection{Alloy composition controlled plasmon tuning}

It has been observed that the compositions of alloy nanoparticles $(\mathrm{Au} / \mathrm{Cu}$ and $\mathrm{Au} / \mathrm{Pt})$ in $\mathrm{SiO}_{2}$ film matrix could be controlled by controlling simply the nominal molar ratios of metal salt precursors in the silica sol (De et al 2000; De and Rao 2003, 2005). This process of doping works well if the respective metal salts remain soluble in the solvent mixture used for the preparation of sols. However, the process failed completely when two metal salts did not remain soluble in the sols. As for example in case of $\mathrm{Au} / \mathrm{Ag}$ system the most commonly available and used precursors are $\mathrm{HAuCl}_{4}$ and $\mathrm{AgNO}_{3}$. These salts are
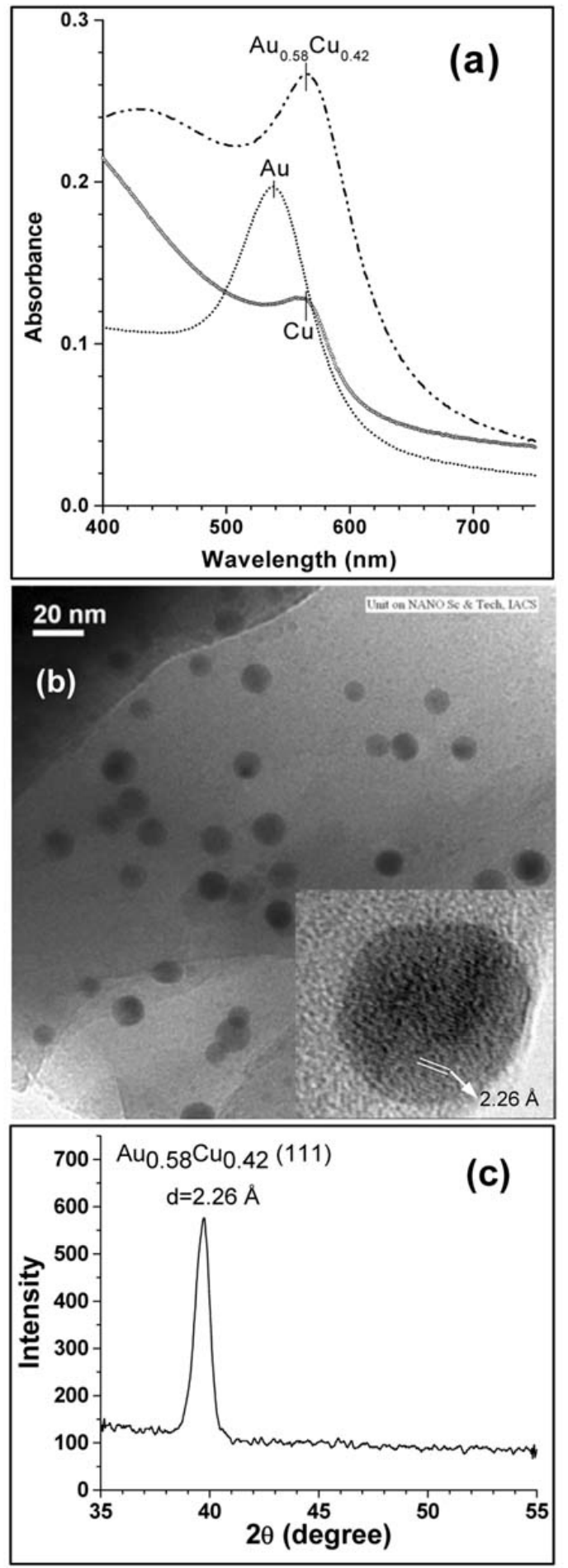

Figure 5. (a) UV-visible spectra of the final heat-treated $\left(750^{\circ} \mathrm{C} / \mathrm{H}_{2}-\mathrm{Ar}\right) 2 \mathrm{~L}$ film containing $\mathrm{Au}_{0.58} \mathrm{Cu}_{0.42}$ nanoparticles and the $\mathrm{Au}$ - and $\mathrm{Cu}$-nanoparticle doped single-layer films, (b) TEM image of $\mathrm{Au}_{0.58} \mathrm{Cu}_{0.42}$ nanoparticle doped TL films obtained after final heat-treatment at $750^{\circ} \mathrm{C}$. Inset of (b) shows the high resolution image and (c) the corresponding XRD spectrum showing only $\mathrm{Au}_{0.58} \mathrm{Cu}_{0.42}\langle 111\rangle$ reflection. (Part of the curves shown in (a) are reproduced from Pal and De 2007b). 
individually soluble in silica sol; however, when they are attempted to mix precipitation of $\mathrm{AgCl}$ resulted instantaneously. We, therefore, developed a new sol-gel 2-layer (2L) protocol (shown schematically in figure 3 ) for the preparation of $\mathrm{Au}-\mathrm{Ag}$ alloy nanoparticles. This strategy involves deposition of two successive overlapping individual layers of $\mathrm{Au}$ - and Ag-doped sols, respectively on glass substrates followed by UV treatment and thermal annealing (Pal and De 2005). The alloy formation with tunable $\mathrm{Au} / \mathrm{Ag}$ compositional control occurred in amorphous glassy matrix through interlayer diffusion of $\mathrm{Ag}$ and $\mathrm{Au}$ in the solid state. This new $2 \mathrm{~L}$ synthetic process also works to prepare other bimetallic alloy nanoparticle (e.g. $\mathrm{Au} / \mathrm{Cu}$ ) doped coatings.

The above synthetic process has the following interesting observations: (i) the alloy nanoparticles are formed through interlayer diffusion controlled mechanism; (ii) the interlayer diffusion is again controlled by the thermal annealing temperature and time; so the formation of intermediate alloy compositions of nanoalloys could be arrested and the product can be isolated; (iii) the desired final nanoalloy particles could also be obtained by controlling the concentrations of individual metals in the respective layers using proper annealing conditions and (iv) the process is suitable for other binary systems. Using this new 2L approach we have synthesized several types of $\mathrm{Au}-\mathrm{Ag}$ nanoalloy incorporated $\mathrm{SiO}_{2}$ films by simply changing the concentration of metals in the individual layers. As, for example, if we intend to prepare $\mathrm{Au}-\mathrm{Ag}(1: 1)$ nanoalloy as a final composition then the individual layers should contain similar molar concentrations of $\mathrm{Au}$ and $\mathrm{Ag}$, and this can be done by applying two successive overlapping coatings of equal thickness containing same equivalent amount (molar) of the two metals. Therefore, the individual coating sols have to be prepared carefully so that the equivalent metal/matrix $\left(\mathrm{SiO}_{2}\right)$ composition and other sol preparation parameters (nature and amount of solvents/ hydrolysis-condensation reactions/room temperature/humidity etc.) remain identical. As an example the synthesis of $\mathrm{Au}-\mathrm{Ag}(1: 1)$ nanoalloy (final composition) incorporated films is described below (Pal and De 2005). First, Audoped coating was deposited on cleaned substrate (here $M_{1}=\mathrm{Au}$ as shown in figure 3 ) and dried at $60^{\circ} \mathrm{C}$ for $30 \mathrm{~min}$ followed by a second coating applied using Agdoped sol (here $M_{2}=\mathrm{Ag}$ as shown in figure 3) onto it. The concentrations of the $\mathrm{Au}$ and $\mathrm{Ag}$ in the respective layers and their individual coating thicknesses are kept similar. The $2 \mathrm{~L}$ coating assembly was again dried at $60^{\circ} \mathrm{C}$ for $30 \mathrm{~min}$ followed by UV treatment. UV-visible spectral analysis of this UV treated film shows peaks at 421 and $542 \mathrm{~nm}$ corresponding to the Ag- and Au-SPR, respectively thus confirming the formation of $\mathrm{Ag}$ and $\mathrm{Au}$ nanoparticles in the individual layers. This $2 \mathrm{~L}$ coating assembly was then heated cumulatively at 450, 500 and $550^{\circ} \mathrm{C}$ in air. The UV-visible spectrum of the respective heat-treated films showed one intense SPR peak in between the $\mathrm{Au}-$ and $\mathrm{Ag}-\mathrm{SPR}$ peak position at 504 $\left(450^{\circ} \mathrm{C} / 1 \mathrm{~h}\right), 497\left(500^{\circ} \mathrm{C} / 1 \mathrm{~h}\right), 480\left(550^{\circ} \mathrm{C} / 1 \mathrm{~h}\right)$ and $468 \mathrm{~nm}$ $\left(550^{\circ} \mathrm{C} / 2 \mathrm{~h}\right)$, respectively due to the formation of $\mathrm{Au}-\mathrm{Ag}$ nanoalloys. The compositions of the intermediate and final nanoalloy are $\mathrm{Au}_{4.3} \mathrm{Ag}_{1}, \mathrm{Au}_{3} \mathrm{Ag}_{1}, \mathrm{Au}_{1.5} \mathrm{Ag}_{1}$ and $\mathrm{Au}_{1} \mathrm{Ag}_{1}$, respectively (Pal and De 2005). It may be mentioned here that the unalloyed $\mathrm{Ag}$ remains as an oxidized species (which is optically non-absorbing) in the intermediate heat-treated films. During heat-treatment, such oxidized silver as well as very small $\mathrm{Au}$ nanoparticles come into contact through interlayer diffusion and form alloy nanoparticles inside the silica films. If the concentrations of $M_{1}$ and $M_{2}$ (figure 3 ) is varied keeping the above final heat-treatment schedule (slight modification may be required on case to case basis), almost any desired $\mathrm{Au}-\mathrm{Ag}$ nanoalloy incorporated films could be prepared using this approach (Pal and De 2007). Figure 4 shows optical absorption spectra of $\mathrm{Au}-\mathrm{Ag}(1: 1)$ alloy nanoparticle incorporated film prepared using the new $2 \mathrm{~L}$ protocol. The spectra of individual single-layer $\mathrm{Au}$ - and Ag-nanoparticle doped films are also shown in figure 4.

This new 2L approach also works well in $\mathrm{Au}-\mathrm{Cu}(1: 1)$ system (Pal and De 2007). In this case heat-treatments were carried out in $\mathrm{H}_{2}-\mathrm{Ar}$ (reducing) atmosphere to reduce the $\mathrm{Cu}$ ions. Here also $\mathrm{Au}-\mathrm{Cu}$ nanoalloy particles of variable atomic ratios were found to be generated as intermediates before the formation of desired final $\mathrm{Au}-\mathrm{Cu}$ alloy nanoparticles. During such annealing conditions, $\mathrm{Au}-\mathrm{Cu}$ nanoalloys of compositions $\mathrm{Au}_{0.84} \mathrm{Cu}_{0.16}, \mathrm{Au}_{0.75} \mathrm{Cu}_{0.25}$ and finally $\mathrm{Au}_{0.58} \mathrm{Cu}_{0.42}$ were formed after heat-treatments at 450,600 and $750^{\circ} \mathrm{C}$, respectively in $\mathrm{H}_{2}-\mathrm{Ar}$ atmosphere. As a result, the UV-visible and XRD analyses of the respective heat-treated films show the shifting of alloy $\mathrm{SPR}$ (in between $\mathrm{Au}$ - and $\mathrm{Cu}-\mathrm{SPR}$ ) and $d$-spacing in accordance with the alloy compositions. TEM and XRD analyses confirm the formation of highly $\langle 111\rangle$ oriented growth of all intermediates and final $\mathrm{Au}-\mathrm{Cu}$ nanoalloy particles inside the $2 \mathrm{~L}$ film matrix. We, therefore, concluded that the alloy formation took place mainly through the diffusion and gradual dissolution of $\mathrm{Cu}$ atoms onto the $\langle 111\rangle$ planes of $\mathrm{Au}$ and intermediate $\mathrm{Au}-\mathrm{Cu}$ nanoalloy crystals. The optical spectra of final heat-treated $2 \mathrm{~L}$ film containing $\mathrm{Au}_{0.58} \mathrm{Cu}_{0.42}$ alloy nanoparticles and single layer $\mathrm{Au}$ - and $\mathrm{Cu}$-nanoparticle doped films are shown in figure 5a. The appearance of an intense alloy-SPR in between $\mathrm{Au}-$ and $\mathrm{Cu}-\mathrm{SPR}$ indicates the formation of $\mathrm{Au}-$ $\mathrm{Cu}$ alloy. TEM study (figure $5 \mathrm{~b}$ ) of this film shows presence of $\langle 111\rangle$ oriented $\mathrm{Au}_{0.58} \mathrm{Cu}_{0.42}$ nanoalloy particles. The high resolution image of one such nanoparticle is shown in the inset of figure $5 \mathrm{~b}$. The corresponding XRD of the film is presented in figure $5 \mathrm{c}$. The appearance of reflections corresponding to only $\langle 111\rangle$ crystalline plane confirms the oriented growth of the nanoalloy particles. The lattice $d$-spacing calculated from the XRD and also from high resolution image corresponds with the composition, $\mathrm{Au}_{0.58} \mathrm{Cu}_{0.42}$ (figures $5 \mathrm{~b}$ and $\mathrm{c}$ ). 
(a)

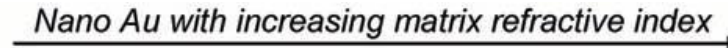

(b)

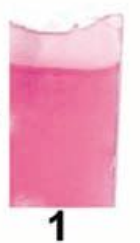

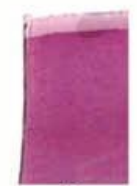

2

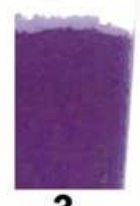

3

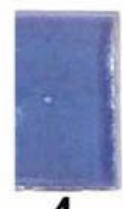

4

AuAg alloy with increasing Ag concentration

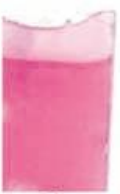

1

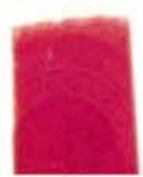

2
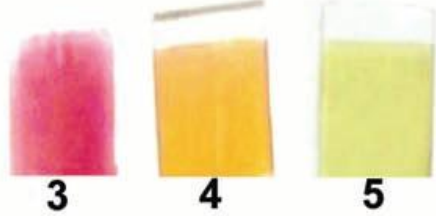

Figure 6. Photographs of (a) $\mathrm{Au}$ nanoparticle doped $\mathrm{SiO}_{2}-$ $\mathrm{TiO}_{2}$ films with refractive index control (see table 1). The $\mathrm{SiO}_{2}: \mathrm{TiO}_{2}$ molar ratios are $1: 0(1), 1: 0.43(2), 1: 1(3), 1: 1.5$ (4) and $1: 2 \cdot 33$ (5), (b) $\mathrm{Au}-\mathrm{Ag}$ alloy nanoparticle doped films with controlled atomic ratios generated by TL strategy. The alloy compositions are: $\mathrm{Au}_{1} \mathrm{Ag}_{0}(1), \mathrm{Au}_{0.81} \mathrm{Ag}_{0.19}$ (2), $\mathrm{Au}_{0.6} \mathrm{Ag}_{0.4}$ (3), $\mathrm{Au}_{0.5} \mathrm{Ag}_{0.5}$ (4) and $\mathrm{Au}_{0} \mathrm{Ag}_{1}$ (5) (Photos marked as 1-4 in (a) are reproduced from Medda et al 2005a).

The nonlinear optical (NLO) characterizations of some of our metal nanoparticle doped films were carried out to show their potential as NLO materials. The films deposited on glass substrates could withstand high intensity laser lights without any damage. The mixed $\mathrm{Ag}-\mathrm{Cu}$ nanoparticle doped $\mathrm{SiO}_{2}$ films (De 1998) showed selfdefocusing nonlinearity and a good NLO absorption leading to the excellent optical limiting (OL) behaviour (Kiran et al 2003, 2004). Au nanoparticle doped $\mathrm{SiO}_{2}-\mathrm{TiO}_{2}$ films having refractive index controlled tunable $\mathrm{Au}-\mathrm{SPR}$ (see \$3.1a) exhibit good optical switching behaviour (Venkatram et al 2006). It has been observed that the refractive indices of the matrices around the $\mathrm{Au}$-nanometals play an important role in modulating good photo switching.

\section{Conclusions}

In this paper we have shown tuning of SPR absorption positions of embedded nanometals inside dielectric matrices leading to the generation of several coloured coatings on glass and plastic substrates. The use of such intense colour generating chromophores in coating matrices can be utilized in a controlled fashion for technological applications. As, for example, photographs of two sets of coloured coatings are presented in figure 6 . In the first set (figure 6a), same size and shaped Au nanoparticles (with similar concentrations) are embedded in dielectric coating matrices $\left(\mathrm{SiO}_{2}-\mathrm{TiO}_{2}\right)$ with different refractive index values (see \$3.1), which causes systematic red-shifting of $\mathrm{Au}-\mathrm{SPR}$ and in the second set (figure $6 \mathrm{~b}$ ) the colour of the film changes due to the presence of $\mathrm{Au}-\mathrm{Ag}$ alloy nanoparticles of different atomic ratios (see §3.2), generated by the new TL protocol. Some of the above metal nanoparticle doped films showed good nonlinear optical (NLO) properties for application in optical limiting and switching.

\section{Acknowledgements}

DST is thankfully acknowledged for supporting this project under the NSTI programme (\# SR/S5/NM-13/2002). Prof. D N Rao and his group, University of Hyderabad, are acknowledged for NLO characterization. The authors thank Director, CGCRI, for encouragement.

\section{References}

De G 1998 J. Sol-Gel Sci. Technol. 11289

De G and Rao C N R 2003 J. Phys. Chem. B107 13597

De G and Rao C N R 2005 J. Mater. Chem. 15891

De G, Tapfer L, Catalano M, Battaglin G, Caccavale F, Gonella F, Mazzoldi P and Haglund R F Jr 1996 Appl. Phys. Lett. 68 3820

De G, Mattei G, Mazzoldi P, Sada C, Battaglin G and Quaranta A 2000 Chem. Mater. 122157

De S and De G 2006a J. Mater. Chem. 163193

De $\mathrm{S}$ and De G 2006b Proc. int. symp. on material chemistry (Mumbai: BARC) pp 419-421

De S, Medda S K and De G 2008 J. Nanosci. Nanotechnol. 8 (paper no. 8jnn09-202)

Jain P K, Eustis S and El-Sayed M A 2006 J. Phys. Chem. B110 18243

Kiran P P, De G and Rao D N 2003 IEE Proc.-Circuits Devices Syst. 150559

Kiran P P, Shivakiran Bhaktha B N, Rao D N and De G 2004 J. Appl. Phys. 966717

Liz-Marzán L M 2006 Langmuir 2232

Mattei G, de Julián Fernández C, Mazzoldi P, Sada C, De G, Battaglin G, Sangregorio C and Gatteschi D 2002 Chem. Mater. 143440

Medda S K, De S and De G 2005a J. Mater. Chem. 153278

Medda S K, Mitra M, De S, Pal S and De G 2005b Pramana-J. Phys. 65931

Mulvaney P 1996 Langmuir 12788

Pal S and De G 2005 Chem. Mater. 176161

Pal S and De G 2007a J. Nanosci. Nanotechnol. 71994

Pal S and De G 2007b J. Mater. Chem. 17493

Sun Y and Xia Y 2003 Analyst 128686

Venkatram N, Kumar R S S, Rao D N, Medda S K, De S and De G 2006 J. Nanosci. Nanotechnol. 61990

Zhou Y, Wang C Y, Zhu Y R and Chen Z Y 1999 Chem. Mater. 112310 